\title{
EXTENDED SOLUTIONS OF THE HARMONIC MAP EQUATION IN THE SPECIAL UNITARY GROUP
}

\author{
NUNO CORREIA AND RUI PACHECO
}

\begin{abstract}
We classify all harmonic maps of finite uniton number from a Riemann surface into $\mathrm{SU}(n)$ in terms of certain pieces of the Bruhat decomposition of $\Omega_{\text {alg }} \mathrm{SU}(n)$, the subgroup of algebraic loops in $\mathrm{SU}(n)$. We give a description of the "Frenet frame data" for such harmonic maps in a given class.
\end{abstract}

\section{INTRODUCTION}

Harmonic maps from a Riemann surface into a Lie group G, with Lie algebra $\mathfrak{g}$, correspond to certain holomorphic maps, the extended solutions, into the group $\Omega \mathrm{G}$ of based smooth loops in $\mathrm{G}$ [14]. If the Fourier series associated to an extended solution $\Phi$ has finitely many terms, we say that $\Phi$ and the corresponding harmonic map have finite uniton number. It is well known that all harmonic maps from the two-sphere have finite uniton number [14].

When $G$ has trivial center, Burstall and Guest [1 have classified harmonic maps with finite uniton number from a Riemann surface $M$ into $\mathrm{G}$ in terms of the pieces of the Bruhat decomposition of

$$
\Omega_{\mathrm{alg}} \mathrm{G}=\left\{\gamma \in \Omega \mathrm{G} \mid \gamma \text { and } \gamma^{-1} \text { have finite Fourier series }\right\} .
$$

More precisely, each piece of the Bruhat decomposition coincides with the unstable manifold associated to the flow of the gradient vector field of a certain Morse-Bott function defined on the Kähler manifold $\Omega_{\mathrm{alg}} \mathrm{G}$; these unstable manifolds are parameterized by the elements of a certain integer lattice $\mathfrak{I}(G)$ in $\mathfrak{g}$; any extended solution with finite uniton number takes values, off a discrete subset of $M$, in one of these unstable manifolds, and so corresponds to some element $\xi \in \mathfrak{I}(\mathrm{G})$; when $G$ has trivial center and maximal torus with dimension $n$, there is a finite subset $\Xi_{\text {can }}(G)$ of the integer lattice $\mathfrak{I}(G)$ with $2^{n}$ elements so that any harmonic map from $M$ to $G$ corresponds to an extended solution with values, off a discrete subset, on the unstable manifold associated to some canonical element $\xi \in \Xi_{\text {can }}(G)$. Among such extended solutions, a distinguished type is that of $\mathrm{S}^{1}$-invariant extended solutions, which correspond to harmonic maps admitting super-horizontal holomorphic lifts into a certain twistor space. For example, all harmonic spheres in $\mathrm{S}^{n}$ and $\mathbb{C P}^{n}$ arise in this way (see [7] and references therein).

In the present paper, we classify all harmonic maps with finite uniton number from $M$ into the special unitary group $\mathrm{SU}(n)$ and corresponding inner symmetric spaces, the Grassmannians

Date: October 8, 2018.

2010 Mathematics Subject Classification. 58E20, 53C43, 53C35.

Key words and phrases. Harmonic maps, extended solutions, special unitary group, finite uniton number.

The authors were partially supported by the Portuguese Government through FCT, under the project PEstOE/MAT/UI0212/2011 (CMUBI). 
$\operatorname{Gr}(k, n)$ of $k$-dimensional subspaces of $\mathbb{C}^{n}$, in terms of certain pieces of the Bruhat decomposition of $\Omega_{\text {alg }} \mathrm{SU}(n)$. For that we use the results of [6] in order to generalize the notion of canonical element of $\mathfrak{I}(\mathrm{G})$ to the case where $G$ has not necessarily trivial center (recall that the center of $\mathrm{SU}(n)$ is isomorphic to the cyclic group $\mathbb{Z}_{n}$ ). Moreover, in the setting of the Grassmannian model for loops groups [11] we give a description of the "Frenet frame data" for such harmonic maps in a given class. The Grassmannian model for loop groups was exploited for the first time in the study of harmonic maps into the unitary group $\mathrm{U}(n)$ by Segal [12]. More recently, the Grassmannian model has been used in the study of harmonic maps into other Lie groups and their inner symmetric spaces [6, 10, 13]. We remark that Ferreira, Simões and Wood [8] established an algebraic formula for all harmonic maps with finite uniton number from a Riemann surface $M$ into the unitary group $\mathrm{U}(n)$ in terms of freely chosen meromorphic functions on $M$ and their derivatives. Since any such harmonic map has constant determinant, this formula can be easily applied in order to obtain all harmonic maps with finite uniton number from $M$ into $\mathrm{SU}(n)$. However, it does not clarifies how to choose the meromorphic functions in order to produce harmonic maps associated to extended solutions in the class of a given element $\xi \in \Xi_{\text {can }}(\mathrm{SU}(n))$. In this paper we shall see how to do that in the case of harmonic maps associated to $\mathrm{S}^{1}$-invariant extended solutions.

\section{Grassmannian MOdel FOR LOOP GROUPS}

Let us start by recalling from Pressley and Segal [11] some standard definitions and facts concerning the Grassmannian model for loop groups.

Fix on $\mathbb{C}^{n}$ the standard complex inner product $\langle\cdot, \cdot\rangle$ and let $e_{1}, \ldots, e_{n}$ be the standard basis vectors for $\mathbb{C}^{n}$. Given a complex subspace $V \subset \mathbb{C}^{n}$, we denote by $\pi_{V}$ the orthogonal projection onto $V$. Let $H^{n}$ be the Hilbert space of square-summable $\mathbb{C}^{n}$-valued functions on the circle and $\langle\cdot, \cdot\rangle_{H}$ the induced complex inner product. This is the closed space generated by the functions $\lambda \mapsto \lambda^{i} e_{j}$, with $i \in \mathbb{Z}$ and $j=1, \ldots, n$. Consider the closed subspace $H_{+}^{n}$ of $H^{n}$ defined by $H_{+}^{n}=\operatorname{Span}\left\{\lambda^{i} e_{j} \mid i \geq 0, j=1, \ldots, n\right\}$. Let $\operatorname{Grass}\left(H^{n}\right)$ denote the set of all closed vector subspaces $W \subset H^{n}$ such that: the projection map $W \rightarrow H_{+}^{n}$ is Fredholm, and the projection map $W \rightarrow H_{+}^{n \perp}$ is Hilbert-Schmidt; the images of the projection maps $W^{\perp} \rightarrow H_{+}^{n}, W \rightarrow H_{+}^{n \perp}$ are contained in $C^{\infty}\left(\mathrm{S}^{1} ; \mathbb{C}^{n}\right)$. Define

$$
\operatorname{Gr}^{n}=\left\{W \in \operatorname{Grass}\left(H^{n}\right) \mid \lambda W \subseteq W\right\} .
$$

The action of the infinite-dimensional Lie group $\Lambda \mathrm{U}(n)=\left\{\gamma: S^{1} \rightarrow \mathrm{U}(n) \mid \gamma\right.$ is smooth $\}$ on $\mathrm{Gr}^{n}$ defined by $\gamma W=\{\gamma f \mid f \in W\}$ is transitive. By considering Fourier series, it is easy to see that the isotropy subgroup at $H_{+}^{n}$ is precisely $\mathrm{U}(n)$. Hence $\mathrm{Gr}^{n} \cong \Lambda \mathrm{U}(n) / \mathrm{U}(n) \cong \Omega \mathrm{U}(n)$. This homogeneous space carries a natural invariant structure of Kähler manifold.

Remark 2.1. Given $W \in \mathrm{Gr}^{n}$, then $\operatorname{dim} W \ominus \lambda W=n$, where $W \ominus \lambda W$ denotes the orthogonal complement of $\lambda W$ in $W$, and the evaluation map ev $: \mathrm{W} \ominus \lambda \mathrm{W}: \rightarrow \mathbb{C}^{\mathrm{n}}$ at $\lambda \in \mathrm{S}^{1}$ is a unitary isomorphism. If we choose an orthonormal basis for $W \ominus \lambda W,\left\{w_{1}, \ldots, w_{n}\right\}$, we can put the vector-valued functions $w_{i}$ side by side to form an $(n \times n)$-matrix valued function $\gamma$ on $\mathrm{S}^{1}$, that is, a loop $\gamma \in \Lambda \mathrm{U}(n)$. It can be shown that $W=\gamma H_{+}^{n}$.

A loop $\gamma \in \Omega \mathrm{U}(n)$ is said to be algebraic if both $\gamma$ and $\gamma^{-1}$ have finite Fourier series. Denote by $\Omega_{\text {alg }} \mathrm{U}(n)$ the subgroup of algebraic loops. This subgroup acts on

$$
\mathrm{Gr}_{\mathrm{alg}}^{n}=\left\{W \in \mathrm{Gr}^{n} \mid \lambda^{k} H_{+}^{n} \subseteq W \subseteq \lambda^{-k} H_{+}^{n} \text { for some } k \in \mathbb{N}\right\}
$$


and we have $\mathrm{Gr}_{\text {alg }}^{n} \cong \Omega_{\text {alg }} \mathrm{U}(n)$. Given $r \leq k$, we set

$$
\Omega_{r}^{k} \mathrm{U}(n)=\left\{\gamma \in \Omega_{\mathrm{alg}} \mathrm{U}(n) \mid \gamma(\lambda)=\sum_{i=r}^{k} \gamma_{i} \lambda^{i}\right\},
$$

where the coefficients $\gamma_{i}$ are constant $(n \times n)$-complex matrices. If $\mathrm{G}$ is a subgroup of $\mathrm{U}(n)$, we shall denote by $\operatorname{Gr}(\mathrm{G})$ the subspace of $\mathrm{Gr}^{n}$ that corresponds to $\Omega \mathrm{G}$ and by $\mathrm{Gr}_{\text {alg }}(\mathrm{G})$ the subspace of $\operatorname{Gr}(\mathrm{G})$ that corresponds to $\Omega_{\mathrm{alg}} \mathrm{G}$.

\section{The Bruhat Decomposition of $\operatorname{Gr}_{\text {alg }}(\mathrm{G})$}

Next we describe the Bruhat decomposition for algebraic loop groups. For more details we refer the reader to [1] and [11].

Consider a compact matrix semi-simple Lie group G. Fix a maximal torus $\mathrm{T}$ of $\mathrm{G}$ with Lie algebra $\mathfrak{t} \subset \mathfrak{g}$, let $\Delta \subset \sqrt{-1} \mathfrak{t}^{*}$ be the corresponding set of roots and denote by $\mathfrak{g}_{\alpha}$ the root space of $\alpha \in \Delta$. The integer lattice $\mathfrak{I}(\mathrm{G})=(2 \pi)^{-1} \exp ^{-1}(e) \cap \mathfrak{t}$ may be identified with the group of homomorphisms $\mathrm{S}^{1} \rightarrow \mathrm{T}$, by associating to $\xi \in \mathfrak{I}(\mathrm{G})$ the homomorphism $\gamma_{\xi}$ defined by $\gamma_{\xi}(\lambda)=$ $\exp (-\sqrt{-1} \ln (\lambda) \xi)$. Let $H_{1}, \ldots, H_{k} \in \mathfrak{t}$ be dual to the positive simple roots $\alpha_{1}, \ldots, \alpha_{k} \in \Delta^{+}$ of $\mathfrak{g}^{\mathbb{C}}: \alpha_{i}\left(H_{j}\right)=\sqrt{-1} \delta_{i j}$. By applying the well-known formula $\operatorname{Ad}(\exp (\eta))=\exp (\operatorname{ad}(\eta))$, for all $\eta \in \mathfrak{g}^{\mathbb{C}}$, we can easily check that the integer lattice $\mathfrak{I}(G)$ is contained in $\mathbb{Z} H_{1} \oplus \ldots \oplus \mathbb{Z} H_{k}$. Denote by $\mathfrak{g}_{i}^{\xi}$ the $\sqrt{-1} i$-eigenspace of ad $\xi$, with $i \in \mathbb{Z}$. We have on $\mathfrak{g}^{\mathbb{C}}$ the structure of graded Lie algebra:

$$
\mathfrak{g}^{\mathbb{C}}=\bigoplus_{i \in\{-r(\xi) \ldots, r(\xi)\}} \mathfrak{g}_{i}^{\xi}, \quad\left[\mathfrak{g}_{i}^{\xi}, \mathfrak{g}_{j}^{\xi}\right] \subset \mathfrak{g}_{i+j}^{\xi},
$$

where $r(\xi)=\max \left\{i \mid \mathfrak{g}_{i}^{\xi} \neq 0\right\}$, and

$$
\mathfrak{g}_{i}^{\xi}=\bigoplus_{\alpha(\xi)=\sqrt{-1} i} \mathfrak{g}_{\alpha}
$$

Set $\Lambda^{+} \mathrm{G}^{\mathbb{C}}=\left\{\gamma: S^{1} \rightarrow \mathrm{G}^{\mathbb{C}} \mid \gamma\right.$ extends holomorphically for $\left.|\lambda| \leq 1\right\}$. For each $\xi \in \mathfrak{I}(\mathrm{G})$, we write $\Omega_{\xi}=\left\{g \gamma_{\xi} g^{-1} \mid g \in \mathrm{G}\right\}$, the conjugacy class of homomorphisms $\mathrm{S}^{1} \rightarrow \mathrm{G}$ which contains $\gamma_{\xi}$. This is a complex homogeneous space:

$$
\Omega_{\xi} \cong \mathrm{G}^{\mathbb{C}} / \mathrm{P}_{\xi}, \text { with } \mathrm{P}_{\xi}=\mathrm{G}^{\mathbb{C}} \cap \gamma_{\xi} \Lambda^{+} \mathrm{G}^{\mathbb{C}} \gamma_{\xi}^{-1} .
$$

Taking account that $\gamma_{\xi} X_{j} \gamma_{\xi}^{-1}=\lambda^{j} X_{j}$ for each $X_{j} \in \mathfrak{g}_{j}^{\xi}$ (this is a direct consequence of the formula $\operatorname{Ad}(\exp (\eta))=\exp (\operatorname{ad}(\eta))$, for all $\left.\eta \in \mathfrak{g}^{\mathbb{C}}\right)$, one can easily check that the Lie algebra of the isotropy subgroup $\mathrm{P}_{\xi}$ is precisely the parabolic subalgebra $\mathfrak{p}_{\xi}=\bigoplus_{i \leq 0} \mathfrak{g}_{i}^{\xi}$ induced by $\xi$.

Now, fix a positive set of roots $\Delta^{+} \subset \Delta$ and set $\mathfrak{I}^{\prime}(\mathrm{G})=\left\{\xi \in \mathfrak{I}(\mathrm{G}) \mid \alpha(\bar{\xi}) \geq 0\right.$ for all $\left.\alpha \in \Delta^{+}\right\}$. We have:

Theorem 3.1. [11] Bruhat decomposition: $\operatorname{Gr}_{\mathrm{alg}}(\mathrm{G})=\bigcup_{\xi \in \mathcal{I}^{\prime}(\mathrm{G})} \Lambda_{\mathrm{alg}}^{+} \mathrm{G}^{\mathbb{C}} \gamma_{\xi} H_{+}^{n}$.

Define $U_{\xi}(\mathrm{G}) \subset \Omega_{\mathrm{alg}} \mathrm{G}$ by $U_{\xi}(\mathrm{G}) H_{+}^{n}=\Lambda_{\text {alg }}^{+} \mathrm{G}^{\mathbb{C}} \gamma_{\xi} H_{+}^{n}$. This is also a complex homogeneous space of $\Lambda_{\text {alg }}^{+} G^{\mathbb{C}}$ with isotropy subgroup at $\gamma_{\xi}$ given by $\Lambda_{\text {alg }}^{+} G^{\mathbb{C}} \cap \gamma_{\xi} \Lambda^{+} G^{\mathbb{C}} \gamma_{\xi}^{-1}$. Moreover, $U_{\xi}(\mathrm{G})$ carries the structure of holomorphic vector bundle over $\Omega_{\xi}$ whose bundle map $u_{\xi}: U_{\xi}(\mathrm{G}) \rightarrow \Omega_{\xi}$ 
is precisely the natural map $[\gamma] \mapsto[\gamma(0)]$. The holomorphic tangent bundle of $U_{\xi}(\mathrm{G})$ is given by

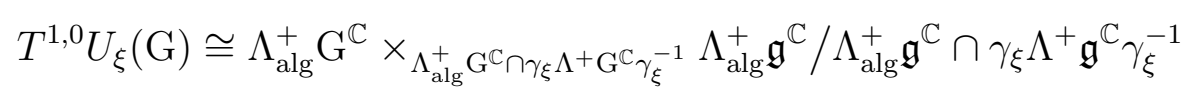

In terms of the Grassmannian model, the bundle map $u_{\xi}: U_{\xi}(\mathrm{G}) \rightarrow \Omega_{\xi}$ can be described as follows. Take $\gamma \in U_{\xi}(\mathrm{G})$ and $W=\gamma H_{+}^{n} \in \mathrm{Gr}_{\text {alg }}(\mathrm{G})$, with $\lambda^{r} H_{+}^{n} \subset W \subset \lambda^{-s} H_{+}^{n}$. Fix $\Psi \in \Lambda_{\text {alg }}^{+} \mathrm{G}^{\mathbb{C}}$ such that $W=\Psi \gamma_{\xi} H_{+}^{n}$. Write

$$
\gamma_{\xi} H_{+}^{n}=\lambda^{-s} A_{-s}^{\xi}+\ldots+\lambda^{r-1} A_{r-1}^{\xi}+\lambda^{r} H_{+}^{n},
$$

where the subspaces $A_{i}^{\xi}$ define a flag

$$
\{0\}=A_{-s-1}^{\xi} \subsetneq A_{-s}^{\xi} \subseteq A_{-s+1}^{\xi} \subseteq \ldots \subseteq A_{r-1}^{\xi} \subsetneq A_{r}^{\xi}=\mathbb{C}^{n} .
$$

Set $A_{i}=\Psi(0) A_{i}^{\xi}=p_{i}\left(W \cap \lambda^{i} H_{+}^{n}\right)$, where $p_{i}: H^{n} \rightarrow \mathbb{C}^{n}$ is defined by

$$
p_{i}\left(\sum \lambda^{j} a_{j}\right)=a_{i}
$$

Then

$$
u_{\xi}(W)=\lambda^{-s} A_{-s}+\ldots+\lambda^{r-1} A_{r-1}+\lambda^{r} H_{+}^{n} .
$$

Following [6], consider the partial order $\preceq$ over $\mathfrak{I}^{\prime}(\mathrm{G})$ defined by: $\xi \preceq \xi^{\prime}$ if $\mathfrak{p}_{i}^{\xi} \subseteq \mathfrak{p}_{i}^{\xi^{\prime}}$ for all $i \geq 0$, where $\mathfrak{p}_{i}^{\xi}=\bigoplus_{j \leq i} \mathfrak{g}_{j}^{\xi}$. Given two elements $\xi, \xi^{\prime} \in \mathfrak{I}^{\prime}(\mathrm{G})$ such that $\xi \preceq \xi^{\prime}$, it can be shown [6] that

$$
\Lambda_{\text {alg }}^{+} \mathrm{G}^{\mathbb{C}} \cap \gamma_{\xi} \Lambda^{+} \mathrm{G}^{\mathbb{C}} \gamma_{\xi}^{-1} \subseteq \Lambda_{\text {alg }}^{+} \mathrm{G}^{\mathbb{C}} \cap \gamma_{\xi^{\prime}} \Lambda^{+} \mathrm{G}^{\mathbb{C}} \gamma_{\xi^{\prime}}^{-1}
$$

This allows us to define, for $\xi \preceq \xi^{\prime}$, a $\Lambda_{\text {alg }}^{+} \mathrm{G}^{\mathbb{C}}$-invariant fibre bundle morphism $\mathcal{U}_{\xi, \xi^{\prime}}: U_{\xi} \rightarrow U_{\xi^{\prime}}$ by

$$
\mathcal{U}_{\xi, \xi^{\prime}}\left(\Psi \gamma_{\xi} H_{+}^{n}\right)=\Psi \gamma_{\xi^{\prime}} H_{+}^{n}, \quad \Psi \in \Lambda_{\text {alg }}^{+} G^{\mathbb{C}} .
$$

Since the holomorphic structures on $U_{\xi}(\mathrm{G})$ and $U_{\xi^{\prime}}(\mathrm{G})$ are induced by the holomorphic structure on $\Lambda_{\text {alg }}^{+} G^{\mathbb{C}}$, the fibre-bundle morphism $\mathcal{U}_{\xi, \xi^{\prime}}$ is holomorphic.

\section{HARMonic MAPs into a Lie Group}

4.1. Extended solutions. Let $M$ be a Riemann surface and $\varphi: M \rightarrow \mathrm{G} \subseteq \mathrm{U}(n)$ a map into a compact matrix Lie group. Equip $\mathrm{G}$ with a bi-invariant metric. Define $\alpha=\varphi^{-1} d \varphi$ and let $\alpha=\alpha^{\prime}+\alpha^{\prime \prime}$ be the type decomposition of $\alpha$ into $(1,0)$ and $(0,1)$-forms. It is well known [14] that $\varphi: M \rightarrow \mathrm{G}$ is harmonic if and only if the loop of 1 -forms given by

$$
\alpha_{\lambda}=\frac{1-\lambda^{-1}}{2} \alpha^{\prime}+\frac{1-\lambda}{2} \alpha^{\prime \prime}
$$

satisfies the Maurer-Cartan equation $d \alpha_{\lambda}+\frac{1}{2}\left[\alpha_{\lambda} \wedge \alpha_{\lambda}\right]=0$ for each $\lambda \in \mathrm{S}^{1}$. Then, if $M$ is simply connected and $\varphi$ is harmonic, we can integrate to obtain a map $\Phi: M \rightarrow \Omega G$, the extended solution associated to $\varphi$, such that $\alpha_{\lambda}=\Phi_{\lambda}^{-1} d \Phi_{\lambda}$ and $\Phi_{-1}=\varphi$. Conversely, if $\Phi: M \rightarrow \Omega \mathrm{G}$ is an extended solution, that is if it integrates a loop of 1-form of the form (6), then $\varphi=\Phi_{-1}: M \rightarrow \mathrm{G}$ is harmonic.

Theorem 4.1. [1] Let $\Phi: M \rightarrow \Omega_{\mathrm{alg}} \mathrm{G}$ be an extended solution. Then there exists some $\xi \in \mathfrak{I}^{\prime}(\mathrm{G})$, and some discrete subset $D$ of $M$, such that $\Phi(M \backslash D) \subseteq U_{\xi}(\mathrm{G})$. 
Given a smooth map $\Phi: M \backslash D \rightarrow U_{\xi}(\mathrm{G})$, consider $\Psi: M \backslash D \rightarrow \Lambda_{\text {alg }}^{+} \mathrm{G}^{\mathbb{C}}$ such that $\Phi H_{+}^{n}=\Psi \gamma_{\xi} H_{+}^{n}$. Clearly, $\Psi \gamma_{\xi}=\Phi b$ for some $b: M \backslash D \rightarrow \Lambda_{\text {alg }}^{+} G^{\mathbb{C}}$. Write

$$
\Psi^{-1} \Psi_{z}=\sum_{i \geq 0} X_{i}^{\prime} \lambda^{i}, \quad \Psi^{-1} \Psi_{\bar{z}}=\sum_{i \geq 0} X_{i}^{\prime \prime} \lambda^{i}
$$

Proposition 4.4 in [1] establishes that $\Phi$ is an extended solution if, and only if,

$$
\operatorname{Im} X_{i}^{\prime} \subset \mathfrak{p}_{i+1}^{\xi}, \quad \operatorname{Im} X_{i}^{\prime \prime} \subset \mathfrak{p}_{i}^{\xi},
$$

where $\mathfrak{p}_{i}^{\xi}=\bigoplus_{j \leq i} \mathfrak{g}_{j}^{\xi}$. The second condition says that $\Phi: M \backslash D \rightarrow U_{\xi}(\mathrm{G})$ is holomorphic.

The bundle morphism $\mathcal{U}_{\xi, \xi^{\prime}}$ and the bundle map $u_{\xi}$ are well behaved with respect to extended solutions:

Theorem 4.2. [6] Given an extended solution $\Phi: M \backslash D \rightarrow U_{\xi}(\mathrm{G})$ and an element $\xi^{\prime} \in \mathfrak{I}^{\prime}(\mathrm{G})$ such that $\xi \preceq \xi^{\prime}$, then $\mathcal{U}_{\xi, \xi^{\prime}}(\Phi)=\mathcal{U}_{\xi, \xi^{\prime}} \circ \Phi: M \backslash D \rightarrow U_{\xi^{\prime}}(\mathrm{G})$ is a new extended solution.

Theorem 4.3. [1] If $\Phi: M \backslash D \rightarrow U_{\xi}(\mathrm{G})$ is an extended solution, then $u_{\xi} \circ \Phi: M \backslash D \rightarrow \Omega_{\xi}$ is an extended solution.

An $\mathrm{S}^{1}$-invariant extended solution is an extended solution which takes values in $\Omega_{\xi}$, for some $\xi \in \mathfrak{I}^{\prime}(\mathrm{G})$.

4.2. Harmonic maps into inner G-symmetric spaces. Given a compact (connected) Lie group $\mathrm{G}$, each connected component of $\sqrt{e}=\left\{g \in \mathrm{G} \mid g^{2}=e\right\}$ is a compact inner symmetric space [1]. Conversely, any compact (connected) inner G-symmetric space may be immersed in $\mathrm{G}$ as a connected component of $\sqrt{e}$. Moreover, the embedding of each component of $\sqrt{e}$ in $\mathrm{G}$ is totally geodesic. Hence harmonic maps into G-inner symmetric spaces can be viewed as special harmonic maps into $\mathrm{G}$.

As in [1, define the involution $\mathcal{I}: \Omega \mathrm{G} \rightarrow \Omega \mathrm{G}$ by $\mathcal{I}(\gamma)(\lambda)=\gamma(-\lambda) \gamma(-1)^{-1}$. Write

$$
\Omega^{\mathcal{I}} \mathrm{G}=\{\gamma \in \Omega \mathrm{G} \mid \mathcal{I}(\gamma)=\gamma\}
$$

for the fixed set of $\mathcal{I}$. Let $M$ be a Riemann surface and $\Phi: M \rightarrow \Omega^{\mathcal{I}} \mathrm{G}$ an extended solution. Then $\varphi=\Phi_{-1}$ defines a harmonic map from $M$ into a connected component of $\sqrt{e}$. Conversely, if $\varphi: M \rightarrow \sqrt{e}$ is a harmonic map, there exists an extended solution $\Phi: M \rightarrow \Omega^{\mathcal{I}} \mathrm{G}$ such that $\varphi=\Phi_{-1}$. Under the identification $\Omega \mathrm{G} \cong \mathrm{Gr}(\mathrm{G}), \mathcal{I}$ induces an involution on $\mathrm{Gr}(\mathrm{G})$, that we shall also denote by $\mathcal{I}$, and $\Omega^{\mathcal{I}} \mathrm{G}$ can be identified with

$$
\operatorname{Gr}^{\mathcal{I}}(\mathrm{G})=\{W \in \operatorname{Gr}(\mathrm{G}) \mid \text { if } s(\lambda) \in W \text { then } s(-\lambda) \in W\} .
$$

Corresponding to the extended solution $\Phi: M \rightarrow \Omega^{\mathcal{I}} \mathrm{G}$, consider $W=\Phi H_{+}: M \rightarrow \mathrm{Gr}^{\mathcal{I}}(\mathrm{G})$.

For each $\xi \in \mathfrak{I}^{\prime}(\mathrm{G})$ we can associate the symmetric space $N_{\xi}=\left\{g \gamma_{\xi}(-1) g^{-1} \mid g \in G\right\}$. If an extended solution takes values in $U_{\xi}^{\mathcal{I}}(\mathrm{G})=U_{\xi}(\mathrm{G}) \cap \Omega^{\mathcal{I}} \mathrm{G}$, then the corresponding harmonic map takes values in $N_{\xi}$. Observe that, for $\xi$ and $\xi^{\prime}$ in $\mathfrak{I}^{\prime}(\mathrm{G})$, if $\xi-\xi^{\prime} \in \mathfrak{I}^{2}(\mathrm{G}):=\pi^{-1} \exp ^{-1}(e) \cap \mathfrak{t}$, then $N_{\xi}=N_{\xi^{\prime}}$. Moreover, as shown in [6], if $\xi \preceq \xi^{\prime}$, then $\mathcal{U}_{\xi, \xi^{\prime}}\left(U_{\xi}^{\mathcal{I}}(\mathrm{G})\right) \subset U_{\xi^{\prime}}^{\mathcal{I}}(\mathrm{G})$. To sum up, if we define a new partial order $\preceq_{\mathcal{I}}$ in $\mathfrak{I}^{\prime}(\mathrm{G})$ by

$$
\xi \preceq_{\mathcal{I}} \xi^{\prime} \quad \text { if } \xi \preceq \xi^{\prime} \text { and } \xi-\xi^{\prime} \in \mathfrak{I}^{2}(\mathrm{G}) \text {, }
$$

the following holds:

Proposition 4.4. If $\xi \preceq_{\mathcal{I}} \xi^{\prime}$, then $\mathcal{U}_{\xi, \xi^{\prime}}\left(U_{\xi}^{\mathcal{I}}(\mathrm{G})\right) \subset U_{\xi^{\prime}}^{\mathcal{I}}(\mathrm{G})$ and $N_{\xi}=N_{\xi^{\prime}}$. 
4.3. Extended solutions from the Grassmannian point of view. Let $W: M \rightarrow \operatorname{Gr}(\mathrm{G})$ correspond to a smooth map $\Phi: M \rightarrow \Omega \mathrm{G}$ under the identification $\Omega \mathrm{G} \cong \mathrm{Gr}(\mathrm{G})$, that is $W=\Phi H_{+}^{n}$. Segal [12] has observed that $\Phi$ is an extended solution if, and only if, $W$ is a solution of equations:

$$
\begin{aligned}
& W_{z} \subseteq \lambda^{-1} W, \\
& W_{\bar{z}} \subseteq W .
\end{aligned}
$$

Condition (8) means that, in any local complex coordinate $z, \frac{\partial s}{\partial z}(z)$ is contained in the subspace $\lambda^{-1} W(z)$ of $H^{n}$, for every (smooth) map $s: M \rightarrow H^{n}$ such that $s(z) \in W(z)$. Inspired by [2] (Section F of Chapter 8), we call (8) the pseudo-horizontality condition. Condition (9) is interpreted in a similar way and states that $W$ is a holomorphic vector subbundle of $M \times H^{n}$.

Remark 4.5. Consider some discrete set $D \subset M$, an element $\xi \in \mathfrak{I}^{\prime}(\mathrm{G})$ and an extended solution $\Phi: M \backslash D \rightarrow U_{\xi}(\mathrm{G})$. As explained in Remark 2.5 of [5], the bundle $W=\Phi H_{+}^{n}$ can be extended holomorphically to $M$, and, consequently, $\Phi$ defines a global extended solution from $M$ to $\Omega_{\text {alg }} \mathrm{G}$.

If $\Phi: M \backslash D \rightarrow U_{\xi}(\mathrm{G})$ is an extended solution and $W=\Phi H_{+}^{n}$, then $u_{\xi}(W)=u_{\xi} \circ \Phi H_{+}^{n}$ is given pointwise by (5) and we get holomorphic subbundles $A_{i}$ of the trivial bundle $\underline{\mathbb{C}}^{n}=M \times \mathbb{C}^{n}$ such that

$$
0 \subsetneq A_{-s} \subseteq \ldots \subseteq A_{r-1} \subsetneq A_{r}=\underline{\mathbb{C}}^{n} .
$$

The pseudo-horizontally condition implies that $A_{i z} \subseteq A_{i+1}$, that is, following again the terminology of [2], the flag of holomorphic vector bundles (10) is super-horizontal.

4.4. Normalization of extended solutions. The following theorem, which is a generalization of Theorem 4.5 in [1], is fundamental to the classification of extended solutions.

Theorem 4.6. [6] Let $\Phi: M \backslash D \rightarrow U_{\xi}(\mathrm{G})$ be an extended solution. Take $\xi^{\prime} \in \mathfrak{I}^{\prime}(\mathrm{G})$ such that $\xi \preceq \xi^{\prime}$ and $\mathfrak{g}_{0}^{\xi}=\mathfrak{g}_{0}^{\xi^{\prime}}$. Then there exists some constant loop $\gamma \in \Omega_{\text {alg }} \mathrm{G}$ such that $\gamma \Phi: M \backslash D \rightarrow U_{\xi^{\prime}}(\mathrm{G})$.

A similar statement holds for extended solutions associated to harmonic maps into symmetric spaces:

Theorem 4.7. Let $\Phi: M \backslash D \rightarrow U_{\xi}^{\mathcal{I}}(\mathrm{G})$ be an extended solution. Take $\xi^{\prime} \in \mathfrak{I}^{\prime}(\mathrm{G})$ such that $\xi \preceq_{\mathcal{I}} \xi^{\prime}$. Then there exists some constant loop $\gamma \in \Omega_{\mathrm{alg}}^{\mathcal{I}} \mathrm{G}$ such that $\gamma \Phi: M \backslash D \rightarrow U_{\xi^{\prime}}^{\mathcal{I}}(\mathrm{G})$.

Proof. We can write $\Phi H_{+}^{n}=\Psi \gamma_{\xi} H_{+}^{n}$, where $\Psi: M \backslash D \rightarrow \Lambda_{\text {alg }}^{+} G^{\mathbb{C}}$ contains only even powers of $\lambda$, and consequently $\Psi^{-1} \Psi_{z}=\sum_{i \geq 0} X_{i}^{\prime} \lambda^{i}$ contains only even powers of $\lambda$. The extended solution equation (17) gives $\operatorname{Im} X_{2 j}^{\prime} \subset \mathfrak{p}_{2 j+1}^{\bar{\xi}}$ for all $j \geq 0$. Set $\hat{\xi}=\xi-\xi^{\prime} \in \mathfrak{I}^{2}(\mathrm{G})$. Clearly $\xi \preceq \hat{\xi}$, hence $\mathfrak{p}_{2 j+1}^{\xi} \subseteq \mathfrak{p}_{2 j+1}^{\hat{\xi}}$ for all $j \geq 0$. On the other hand, since $\alpha(\hat{\xi})=2 \sqrt{-1} \mathbb{Z}$ for any positive root $\alpha$, we have $\mathfrak{g}_{2 j+1}^{\hat{\xi}}=0$ and, consequently, $\mathfrak{p}_{2 j+1}^{\hat{\xi}}=\mathfrak{p}_{2 j}^{\hat{\xi}}$. Hence,

$$
\operatorname{Im} \Psi^{-1} \Psi_{z} \subseteq \bigoplus_{j \geq 0} \mathfrak{p}_{2 j+1}^{\xi} \lambda^{2 j} \subseteq \bigoplus_{j \geq 0} \mathfrak{p}_{2 j}^{\hat{\xi}} \lambda^{2 j} \subseteq \Lambda_{\text {alg }}^{+} \mathfrak{g}^{\mathbb{C}} \cap \gamma_{\hat{\xi}} \Lambda^{+} \mathfrak{g}^{\mathbb{C}} \gamma_{\hat{\xi}}^{-1}
$$

Taking account (2), we conclude that $\mathcal{U}_{\xi, \hat{\xi}}(\Phi)$ is anti-holomorphic. On the other hand, since any extended solution is holomorphic and $\Phi$ is an extended solution, Theorem 4.2 asserts that 
$\mathcal{U}_{\xi, \hat{\xi}}(\Phi)$ is also holomorphic. Being both holomorphic and anti-holomorphic, it must be equal to a constant loop $\gamma^{-1}$. By Proposition 4.4 we have $\gamma^{-1} \in \Omega_{\text {alg }}^{\mathcal{I}}$ G. Write $\Psi \gamma_{\hat{\xi}}=\gamma^{-1} b$, for some map $b: M \rightarrow \Lambda^{+} \mathrm{G}$. Then

$$
\Phi H_{+}^{n}=\Psi \gamma_{\xi} H_{+}^{n}=\gamma^{-1} b \gamma_{\hat{\xi}}^{-1} \gamma_{\xi} H_{+}^{n}=\gamma^{-1} b \gamma_{\xi^{\prime}} H_{+}^{n},
$$

which implies that $\gamma \Phi$ takes values in $U_{\xi^{\prime}}^{\mathcal{I}}(\mathrm{G})$.

Given $\xi=\sum n_{i} H_{i}$ and $\xi^{\prime}=\sum n_{i}^{\prime} H_{i}$ in $\mathfrak{I}^{\prime}(\mathrm{G})$, we have $n_{i}, n_{i}^{\prime} \geq 0$ and observe that $\xi \preceq \xi^{\prime}$ if and only if $n_{i}^{\prime} \leq n_{i}$ for all $i$. For each $I \subseteq\{1, \ldots, k\}$, define the cone

$$
\mathfrak{C}_{I}=\left\{\sum_{i=1}^{k} n_{i} H_{i} \mid n_{i} \geq 0, n_{j}=0 \text { iff } j \notin I\right\} .
$$

Definition 4.8. Let $\xi \in \mathfrak{I}^{\prime}(\mathrm{G}) \cap \mathfrak{C}_{I}$. We say that $\xi$ is a $I$-canonical element of $\mathfrak{g}$ with respect to $\Delta^{+}$if it is a maximal element of $\left(\mathfrak{I}^{\prime}(\mathrm{G}) \cap \mathfrak{C}_{I}, \preceq\right)$, that is, if $\xi \preceq \xi^{\prime}$ and $\xi^{\prime} \in \mathfrak{I}^{\prime}(\mathrm{G}) \cap \mathfrak{C}_{I}$ then $\xi=\xi^{\prime}$. Similarly, we say that $\xi$ is a symmetric canonical element of $\mathfrak{g}$ with respect to $\Delta^{+}$if it is a maximal element of $\left(\mathfrak{I}^{\prime}(\mathrm{G}), \preceq_{\mathcal{I}}\right)$

When $G$ has trivial center, which is the case considered in [1], the duals $H_{1}, \ldots, H_{k}$ belong to the integer lattice. Then, for each $I$ there exists a unique $I$-canonical element, which is given by $\xi_{I}=\sum_{i \in I} H_{i}$.

Theorem 4.9. Let $\Phi: M \rightarrow \Omega_{\mathrm{alg}} \mathrm{G}$ be an extended solution. There exist a constant loop $\gamma \in \Omega_{\mathrm{alg}} \mathrm{G}$, a subset $I \subseteq\{1, \ldots, k\}$, a I-canonical element $\xi^{\prime}$ and a discrete subset $D \subset M$, such that $\gamma \Phi(M \backslash D) \subseteq U_{\xi^{\prime}}(\mathrm{G})$.

Proof. Take $D \subset M$ and $\xi \in \mathfrak{I}^{\prime}(\mathrm{G})$ in the conditions of Theorem 4.1. Write $\xi=\sum_{i=1}^{k} n_{i} H_{i}$, with $n_{i} \geq 0$, and set $I=\left\{i \mid n_{i}>0\right\}$. By Zorn's lemma, there certainly exists a $I$-canonical element $\xi^{\prime}$ such that $\xi \preceq \xi^{\prime}$. On the other hand, from (1) we see that $\mathfrak{g}_{0}^{\xi}=\mathfrak{g}_{0}^{\xi^{\prime}}$. Hence the result follows from Theorem 4.6.

Theorem 4.10. Let $\Phi: M \rightarrow \Omega_{\text {alg }}^{\mathcal{I}} \mathrm{G}$ be an extended solution with values in $U_{\xi}^{\mathcal{I}}(\mathrm{G})$, for some $\xi \in \mathfrak{I}^{\prime}(\mathrm{G})$, off a discrete set $D$. There exist a constant loop $\gamma \in \Omega_{\mathrm{alg}}^{\mathcal{I}} \mathrm{G}$ and a symmetric canonical element $\xi^{\prime}$ such that $\gamma \Phi(M \backslash D) \subseteq U_{\xi^{\prime}}^{\mathcal{I}}(\mathrm{G})$ and $N_{\xi}=N_{\xi^{\prime}}$.

Proof. By Zorn's lemma, there certainly exists a symmetric canonical element $\xi^{\prime}$ such that $\xi \preceq_{\mathcal{I}} \xi^{\prime}$. The result follows from Proposition 4.4 and Theorem 4.7 .

4.5. Frenet frame data for extended solutions into $\Omega_{\mathrm{alg}} \mathrm{U}(n)$. Given a finite collection $\left\{s_{j}\right\}$ of meromorphic sections of the trivial bundle $\mathbb{C}^{n}=M \times \mathbb{C}^{n}$, we obtain an holomorphic bundle away from a discrete subset of $M$, and we can fill in holes to extend it to subbundle $E$ over $M$ of $\underline{\mathbb{C}}^{n}$. In this case, we denote $E=\operatorname{Span}\left\{s_{j}\right\}$. Reciprocally, any holomorphic subbundle $E$ of $\underline{\mathbb{C}}^{n}$ has a global meromorphic frame $\left\{s_{1}, \ldots, s_{k}\right\}$, with $k=\operatorname{rank} E$, as explained in [13]. For $i>0$, the $(i)$-th osculating bundle of $E$ is the subbundle $E^{(i)}$ of $\underline{\mathbb{C}}^{n}$ spanned by the local holomorphic sections of $E$ and their derivatives up to $i$. We also define the $(-i)$-th osculating bundle of $E$ as the subbundle $E^{(-i)}$ of $\underline{\mathbb{C}}^{n}$ spanned by the local holomorphic sections of $E$ whose derivatives up to $i$ are also local sections of $E$. Let $g_{E}=\operatorname{rank} E^{(1)}-\operatorname{rank} E$ and $r_{E}$ be the remainder of the positive integer division of $\operatorname{rank} E$ by $g_{E}: \operatorname{rank} E=q_{E} g_{E}+r_{E}$. 
As Guest [9] has observed, any smooth map $W: M \rightarrow \mathrm{Gr}^{n}$ corresponding to an extended solution $\Phi: M \rightarrow \Omega_{r}^{k} \mathrm{U}(n)$ is generated by a certain holomorphic subbundle $X$, a Frenet frame of $\Phi$, of the trivial bundle $M \times \lambda^{r} H_{+} / \lambda^{k} H_{+}$by setting

$$
W=X+\lambda X^{(1)}+\ldots+\lambda^{k-r-1} X^{(k-r-1)}+\lambda^{k} H_{+} .
$$

Hence any extended solution $\Phi: M \rightarrow \Omega_{\mathrm{alg}} \mathrm{U}(n)$ can be obtained by applying a finite number of algebraic operations on sets of meromorphic functions on $M$, since $X$ can be chosen arbitrarily. In [8, 13] the authors established explicit algebraic formulae relating Frenet frames $X$ with different classes of uniton factorizations of harmonic maps. Next we will give a description of the Frenet frames associated to extended solutions with values in a fixed piece $U_{\xi}(\mathrm{U}(n))$ of the Bruhat decomposition of $\Omega_{\mathrm{alg}} \mathrm{U}(n)$ and we establish a pure algebraic method to obtain all $\mathrm{S}^{1}$-invariant extended solutions with values in a fixed $\Omega_{\xi}$.

Choose a local complex coordinate $z$ and a local section $s$ of $E$. Differentiating $\pi_{E}^{\perp}(s)=$ 0 , where $\pi_{E}^{\perp}$ is the orthogonal projection onto $E^{\perp}$, we get $\pi_{E}^{\perp}\left(s_{z}\right)=-\left(\pi_{E}^{\perp}\right)_{z}(s)$. Hence the association $s \mapsto \pi_{E}^{\perp}\left(s_{z}\right)$ defines a local vector bundle morphism $\mathcal{A}_{E}: E \rightarrow E^{\perp}$, which, following [3], we call the $\partial^{\prime}$-second fundamental form of $E$ in $\mathbb{C}^{n}$, whose kernel and image do not depend on the choice of the local coordinate $z$. It follows from the linearity of the $\partial^{\prime}$-second fundamental form that:

Lemma 4.11. Let $E$ be a holomorphic vector subbundle of $\underline{\mathbb{C}}^{n}$.

a) For all $i \geq 1, E^{(-i)}=\operatorname{ker} \mathcal{A}_{E^{(-i+1)}}$ is locally spanned by those sections $s$ of $E$ solving the following system of algebraic linear equations: $\left(\pi_{E^{(-j)}}^{\perp}\right)_{z}(s)=0$ for all $j=0, \ldots, i-1$;

b) $g_{E^{(i)}} \leq g_{E}$ and $\operatorname{rank} E^{(i)} \leq \operatorname{rank} E+i g_{E}$ for all $i \geq 1$ (the equalities hold for $i=1$ );

c) $g_{E^{(-i)}} \leq g_{E}$ and $\operatorname{rank} E^{(-i)} \geq \operatorname{rank} E-i g_{E}$ for all $i \geq 1$ (the equalities hold for $i=1$ );

d) For each $g \geq g_{E}$, there exists a super-horizontal flag of holomorphic subbundles

$$
E_{-q} \subsetneq E_{-q+1} \subsetneq \ldots \subsetneq E_{-1} \subsetneq E_{0}=E,
$$

such that rank $E_{-i}=\operatorname{rank} E-i g$, where the integer $q \leq q_{E}$ is the quotient of the positive integer division of rank $E$ by $g$ : $\operatorname{rank} E=q g+r$.

Proof. For example, since $E^{(-i-1)}=\operatorname{ker} \mathcal{A}_{E^{(-i)}}$, we have, for all $i \geq 0$,

$$
g_{E^{(-i)}}=\operatorname{rank} \operatorname{im} \mathcal{A}_{E^{(-i)}}=\operatorname{rank} \operatorname{coim} \mathcal{A}_{E^{(-i)}}=\operatorname{rank} E^{(-i)}-\operatorname{rank} E^{(-i-1)} .
$$

On the other hand, since the image of $\mathcal{A}_{E^{(-i-1)}}$ is contained in $E^{(-i)} \ominus E^{(-i-1)}$, for all $i \geq 0$, we also have $g_{E^{(-i-1)}} \leq \operatorname{rank} E^{(-i)}-\operatorname{rank} E^{(-i-1)}$. Hence, for all $i \geq 0, g_{E^{(-i)}} \leq g_{E}$.

To construct a flag (12), start by taking an arbitrary holomorphic subbundle $E_{-1} \subseteq E^{(-1)}$ with $\operatorname{rank} E_{-1}=\operatorname{rank} E-g \leq \operatorname{rank} E-g_{E}=\operatorname{rank} E^{(-1)}$. Clearly,

$$
g_{E_{-1}}=\operatorname{rank} E_{-1}^{(1)}-\operatorname{rank} E_{-1} \leq \operatorname{rank} E-\operatorname{rank} E_{-1}=g .
$$

Hence

$$
\operatorname{rank} E_{-1}^{(-1)}=\operatorname{rank} E_{-1}-g_{E_{-1}} \geq \operatorname{rank} E-2 g,
$$

and we see that there exists a holomorphic subbundle $E_{-2}$ of $E_{-1}^{(-1)}$ with rank $E_{-2}=$ rank $E-2 g$. Proceeding recursively we find after $q$ steps a super-horizontal flag of holomorphic subbundles (12).

The following construction is fundamental for our purposes: 
Lemma 4.12. Let $T \subset E$ be two holomorphic subbundles of $\underline{\mathbb{C}}^{n}$. Fix a positive integer $g$, with $g \geq \max \left\{g_{T}, g_{E}\right\}$, and assume that, for some $i, j \geq 0$, we have $T^{(j)} \subset E^{(-i)}$. Given an integer $d$ with $\operatorname{rank} T^{(j)}<d<\operatorname{rank} E^{(-i)}$, any holomorphic subbundle $F$ satisfying $T^{(j)} \subset F \subset E^{(-i)}$, rank $F=d$, and $g_{F} \leq g$, arises as follows:

a) Set $k_{0}=\max \left\{k \mid d-k g>\operatorname{rank} T^{(j-k)}\right\}$ and $r_{0}=d-k_{0} g-\operatorname{rank} T^{\left(j-k_{0}\right)}$. Choose $r_{0}$ linearly independent meromorphic sections $s_{1}, \ldots, s_{r_{0}}$ of $E^{\left(-i-k_{0}\right)}$ so that the holomorphic vector bundle

$$
F_{-k_{0}}=T^{\left(j-k_{0}\right)}+\operatorname{Span}\left\{s_{1}, \ldots, s_{r_{0}}\right\}
$$

has rank $d-k_{0} g$. Independently of the choice of these meromorphic sections, we have $g_{F_{-k_{0}}} \leq g$.

b) Choose $r_{1}=d-\left(k_{0}-1\right) g-\operatorname{rank} F_{-k_{0}}^{(1)}$ meromorphic sections $s_{r_{0}+1}, \ldots, s_{r_{0}+r_{1}}$ of $E^{\left(-i-k_{0}+1\right)}$ so that the holomorphic vector subbundle

$$
F_{-k_{0}+1}=F_{-k_{0}}^{(1)}+\operatorname{Span}\left\{s_{r_{0}+1}, \ldots, s_{r_{0}+r_{1}}\right\}
$$

has rank $d-\left(k_{0}-1\right) g$. We have $g_{F_{-k_{0}+1}} \leq g$.

c) Repeat this procedure $k_{0}$ times to find a super-horizontal flag of holomorphic subbundles $F_{-k_{0}} \subsetneq \ldots \subsetneq F_{-1} \subsetneq F_{0}=F$, with

$$
\begin{array}{r}
F_{-k_{0}+l}=F_{-k_{0}+l-1}^{(1)}+\operatorname{Span}\left\{s_{r_{0}+\ldots+r_{l-1}+1}, \ldots, s_{r_{0}+\ldots+r_{l-1}+r_{l}}\right\}, \\
r_{l}=d-\left(k_{0}-l\right) g-\operatorname{rank} F_{-k_{0}+l-1}^{(1)} \text { and } \operatorname{rank} F_{-k_{0}+l}=d-\left(k_{0}-l\right) g .
\end{array}
$$

Proof. Since $d<\operatorname{rank} E^{(-i)}$ and $g_{E^{(-i)}} \leq g_{E} \leq g$, by Lemma 4.11 inequalities

$$
d-k g<\operatorname{rank} E^{(-i)}-k g_{E^{(-i)}} \leq \operatorname{rank} E^{(-i-k)}
$$

hold for each $k \geq 0$. Hence we can always take $r_{0} \geq 0$ linearly independent meromorphic sections of $E^{\left(-i-k_{0}\right)}$ so that $F_{-k_{0}}$ defined by (14) has rank $d-k_{0} g$. We have to check now that $g_{F_{-k_{0}}} \leq g$. By definition of $k_{0}$ we have $d-\left(k_{0}+1\right) g \leq \operatorname{rank} T^{\left(j-k_{0}-1\right)}$. Then,

$$
\begin{aligned}
g_{F_{-k_{0}}} & \leq g_{T^{\left(j-k_{0}\right)}}+r_{0}=g_{T^{\left(j-k_{0}\right)}}+d-k_{0} g-\operatorname{rank} T^{\left(j-k_{0}\right)} \\
& \leq g_{T^{\left(j-k_{0}\right)}}+g-\left(\operatorname{rank} T^{\left(j-k_{0}\right)}-\operatorname{rank} T^{\left(j-k_{0}-1\right)}\right)=g_{T^{\left(j-k_{0}\right)}}+g-g_{T^{\left(j-k_{0}\right)}}=g .
\end{aligned}
$$

Since $F_{-k_{0}} \subseteq \operatorname{ker} \mathcal{A}_{F_{-k_{0}+1}}$, then $g_{F_{-k_{0}+1}} \leq \operatorname{rank} F_{-k_{0}+1}-\operatorname{rank} F_{-k_{0}}=g$. On the other hand, it is clear that $r_{1} \geq 0$. Hence the construction of item $\mathrm{b}$ ) is possible and we can proceed recursively until find a super-horizontal flag of holomorphic subbundles $F_{-k_{0}} \subsetneq \ldots \subsetneq F_{-1} \subsetneq F_{0}=F$, with $F_{-k_{0}+l}$ given by (15), where $F$ is certainly in the required conditions.

Reciprocally, any $F$ as required certainly arises in this way. In fact, by Lemma 4.11 there always exists a super-horizontal flag of holomorphic subbundles $F_{-q} \subsetneq \ldots \subsetneq F_{-k_{0}} \subsetneq \ldots \subsetneq$ $F_{-1} \subsetneq F_{0}=F$, with $k_{0}=\max \left\{k \mid d-k g>\operatorname{rank} T^{(j-k)}\right\}$. We can choose such sequence so that $T^{\left(j-k_{0}\right)} \subsetneq F_{-k_{0}}$.

Now we are in conditions to establish an algorithm to obtain all $\mathrm{S}^{1}$-invariant extended solutions with values in a given $\Omega_{\xi}$. 
Theorem 4.13. Fix $\xi \in \mathfrak{I}(\mathrm{U}(n))$ and consider the corresponding flag (3). Set $d_{i}=\operatorname{dim} A_{i}^{\xi}$ and $h_{i}=d_{i+1}-d_{i}$. Any super-horizontal flag of holomorphic vector subbundles

$$
\{0\}=A_{-r-1} \subsetneq A_{-r} \subseteq \ldots \subseteq A_{k-1} \subsetneq A_{k}=\underline{\mathbb{C}}^{n}
$$

with $\operatorname{rank} A_{i}=d_{i}$ arises as follows:

a) Set $l=\min \left\{h_{i} \mid i=-r-1, \ldots, k-1\right\}$ and $m=\max \left\{i \mid l=h_{i}\right\}$. Apply Lemma 4.12 (with $T=\{0\}, E=\underline{\mathbb{C}}^{n}, d=d_{m}$ and $g=l$ ) to find $A_{m}$.

b) Set $l_{1}=\min \left\{h_{i} \mid-r-1 \leq i<m\right\}, m_{1}=\max \left\{i \mid l_{1}=h_{i},-r-1 \leq i<m\right\}$. Apply Lemma 4.12 (with $T=\{0\}, E=A_{m}, d=d_{m_{1}}$ and $g=l_{1}$ ) to find $A_{m_{1}} \subseteq A_{m}^{\left(m_{1}-m\right)}$.

c) Set $l_{\hat{1}}=\min \left\{h_{i} \mid m<i \leq k-1\right\}$ and $m_{\hat{1}}=\max \left\{i \mid l_{\hat{1}}=h_{i}, m<i \leq k-1\right\}$, and apply Lemma 4.12 (with $T=A_{m}, E=\underline{\mathbb{C}}^{n}, d=d_{m_{\hat{1}}}$ and $g=l_{\hat{1}}$ ) to find $A_{m_{\hat{1}}} \supseteq A_{m}^{\left(m_{\hat{1}}-m\right)}$.

d) Proceed recursively until obtain a flag of the form (16).

Remark 4.14. In [1], the authors introduce a method to obtain super-horizontal flags of holomorphic subspaces associated to a given element $\xi \in \mathfrak{I}^{\prime}(G)$. However, their method involves integration of meromorphic functions.

Finally, take a super-horizontal flag of holomorphic vector subbundles (16) and the corresponding $\mathrm{S}^{1}$-invariant extended solution $W_{A}$. Take a meromorphic frame $s_{1}, \ldots, s_{d_{k-1}}$ of $A_{k-1}$ such that, for each $i \in\{-r, \ldots, k-1\}, s_{1}, \ldots, s_{d_{i}}$ is a meromorphic frame of $A_{i}$ and $s_{1}, \ldots, s_{d_{i}}, s_{d_{i}+1}, \ldots, s_{d_{i}+g_{i}}$ is a meromorphic frame of $A_{i}^{(1)}$. The extended solution $W$, with values in $U_{\xi}(\mathrm{U}(n))$ and $u_{\xi}(W)=W_{A}$, have Frenet frames of the form

$$
\begin{aligned}
X & =\operatorname{Span}\left\{s_{1} \lambda^{-r}+w_{1} \lambda^{-r+1}, \ldots, s_{d_{-r}} \lambda^{-r}+w_{d_{-r}} \lambda^{-r+1}\right\} \\
& +\sum_{i=-r}^{k-2} \operatorname{Span}\left\{s_{d_{i}+g_{i}+1} \lambda^{i+1}+w_{d_{i}+g_{i}+1} \lambda^{i+2}, \ldots, s_{d_{i+1}} \lambda^{i+1}+w_{d_{i+1}} \lambda^{i+2}\right\} ;
\end{aligned}
$$

where, for each $j \in\left\{1, \ldots, d_{k-1}\right\}, w_{j}$ is a meromorphic section of $M \times H_{+}^{n} / \lambda^{r+k} H_{+}^{n}$. However, in the general case, these meromorphic sections $w_{j}$ can not be chosen arbitrarily. For example, if $s_{1}$ is a constant section, $w_{1}^{\prime} \lambda^{-r+2}$ becomes a section of $W$. So we have to impose that $p_{0}\left(w_{1}^{\prime}\right)$, whith $p_{0}$ the projection defined by (4), has no orthogonal component onto $A_{-r+2}^{\perp}$. In sections 5.4 and 5.5 we shall discuss in detail some examples.

\section{ExTEndED SOLUTiONS IN $\Omega$ SU $(n)$}

5.1. Grassmannian model for $\Omega S U(n)$. Consider the exterior product $\wedge$ of vectors in $\mathbb{C}^{n}$ and extend it to $H^{n}$ as follows: if $f, g \in H^{n}$, then $(f \wedge g)(\lambda)=f(\lambda) \wedge g(\lambda)$. The loop group $\Omega \mathrm{U}(n)$ acts on $\wedge^{n} H^{n}$ in the natural way:

$$
\gamma\left(f_{1} \wedge \ldots \wedge f_{n}\right):=\gamma f_{1} \wedge \ldots \wedge \gamma f_{n}=\operatorname{det}(\gamma)\left(f_{1} \wedge \ldots \wedge f_{n}\right) .
$$

The Grassmannian model of $\Omega \mathrm{SU}(n)$ is given by:

Proposition 5.1. A subspace $W \in \mathrm{Gr}^{n}$ corresponds to a loop in $\mathrm{SU}(n)$ if, and only if, it belongs to

$$
\operatorname{Gr}(\mathrm{SU}(n))=\left\{W \in \mathrm{Gr}^{n} \mid \wedge^{n} W=\wedge^{n} H_{+}^{n}\right\}
$$


Proof. If $\gamma \in \Omega \mathrm{SU}(n)$, then it is clear that $\wedge^{n} W=\wedge^{n} H_{+}^{n}$, since $\Omega \mathrm{SU}(n)$ acts trivially on the $\wedge^{n} H^{n}$. Conversely, suppose that $\wedge^{n} W=\wedge^{n} H_{+}^{n}$. For each $\lambda \in \mathrm{S}^{1}$, consider the isomorphism given by the evaluation map at $\lambda, \mathrm{ev}_{\lambda}: W \ominus \lambda W \rightarrow \mathbb{C}^{n}$. Set $\gamma(\lambda)=\mathrm{ev}_{\lambda} \circ \mathrm{ev}_{1}^{-1}$, which is a loop of $\Omega \mathrm{U}(n)$ and, by Remark 2.1, verifies $W=\gamma H_{+}^{n}$. By hypothesis $\wedge^{n}(W \ominus \lambda W) \subset \wedge^{n} H_{+}^{n}$. Hence, $\mathrm{ev}_{1}^{-1}\left(e_{1}\right) \wedge \ldots \wedge \mathrm{ev}_{1}^{-1}\left(e_{n}\right) \in \wedge^{n} H_{+}^{n}$. Since

$$
\begin{aligned}
\operatorname{det}(\gamma)\left(e_{1} \wedge \ldots \wedge e_{n}\right) & =\gamma\left(e_{1}\right) \wedge \gamma\left(e_{2}\right) \wedge \ldots \wedge \gamma\left(e_{n}\right)=\mathrm{ev}_{\lambda} \circ \mathrm{ev}_{1}^{-1}\left(e_{1}\right) \wedge \ldots \wedge \mathrm{ev}_{\lambda} \circ \mathrm{ev}_{1}^{-1}\left(e_{n}\right) \\
& =\operatorname{ev}_{\lambda}\left(\mathrm{ev}_{1}^{-1}\left(e_{1}\right) \wedge \ldots \wedge \mathrm{ev}_{1}^{-1}\left(e_{n}\right)\right)
\end{aligned}
$$

it follows that $\operatorname{det}(\gamma)$ is in $H_{+}^{1}$.

Now, since $\wedge^{n} \gamma H_{+}^{n}=\wedge^{n} H_{+}^{n}$, we also have $\wedge^{n} H_{+}^{n}=\wedge^{n} \gamma^{-1} H_{+}^{n}$. Hence, by the same argument as above, $\operatorname{det}(\gamma)^{-1}$ is in $H_{+}^{1}$. On the other hand, the fact that $\gamma$ takes values in $\mathrm{U}(n)$ implies that $\operatorname{det}(\gamma)^{-1}=\overline{\operatorname{det}(\gamma)}$, which means that $\operatorname{det}(\gamma)^{-1}$ is also in $H_{-}^{1}$. This is possible if and only if $\operatorname{det}(\gamma)$ is constant. Since $\gamma(1)=e$, we must have $\operatorname{det}(\gamma)=1$.

Proposition 5.2. If $\xi \in \mathfrak{I}(\mathrm{SU}(n)) \subset \mathfrak{I}(\mathrm{U}(n))$, then $U_{\xi}(\mathrm{SU}(n))=U_{\xi}(\mathrm{U}(n))$.

Proof. Let $\gamma \in U_{\xi}(\mathrm{U}(n))$. Then $\gamma H_{+}^{n}=\Psi \gamma_{\xi} H_{+}^{n}$ for some $\Psi \in \Lambda_{\text {alg }}^{+} \mathrm{U}(n)$. Hence

$$
\wedge^{n} \gamma H_{+}^{n}=\wedge^{n} \Psi \gamma_{\xi} H_{+}^{n}=\wedge^{n} \Psi W_{\xi}=\operatorname{det}(\Psi) \wedge^{n} W_{\xi}=\operatorname{det}(\Psi) \wedge^{n} H_{+}^{n} .
$$

Since $\Psi \in \Lambda_{\text {alg }}^{+} \mathrm{U}(n), \operatorname{det}(\Psi)$ is polynomial in $\lambda$, hence $\wedge^{n} \gamma H_{+}^{n} \subseteq \wedge^{n} H_{+}^{n}$. Conversely, from (18) we see that

$$
\wedge^{n} H_{+}^{n}=\operatorname{det}\left(\Psi^{-1}\right) \wedge^{n} \gamma H_{+}^{n}
$$

and since $\operatorname{det}\left(\Psi^{-1}\right)$ is also polynomial in $\lambda$, we conclude that $\wedge^{n} H_{+}^{n} \subseteq \wedge^{n} \gamma H_{+}^{n}$.

In particular, if $\xi \in \mathfrak{I}(\mathrm{SU}(n))$, all the extended solutions $W: M \backslash D \rightarrow U_{\xi}(\mathrm{SU}(n))$ arise from a Frenet frame of the form (17) without any further restriction on the choice of the meromorphic data.

5.2. Canonical elements of $\mathrm{SU}(n)$. Let $E_{i}$ be the $(n \times n)$-matrix whose $(i, i)$ entry is $\sqrt{-1}$ and whose other entries are all 0 . The algebra of diagonal matrices

$$
\mathfrak{t}^{\mathbb{C}}=\left\{\sum a_{i} E_{i}: a_{i} \in \mathbb{C}, \sum a_{i}=0\right\}
$$

is a Cartan subalgebra of $\mathfrak{s u}(n)^{\mathbb{C}}=\mathfrak{s l}(n, \mathbb{C})$. Let $L_{i}$ in the dual of $\mathfrak{t}^{\mathbb{C}}$ be defined by $L_{i}\left(\sum a_{j} E_{j}\right)=$ $\sqrt{-1} a_{i}$. The corresponding set of roots $\Delta \in \sqrt{-1} \mathfrak{t}^{*}$ is given by $\Delta=\left\{L_{i}-L_{j}: i, j=1, \ldots, n\right\}$ and $\Delta^{+}=\left\{L_{i}-L_{j}: i<j\right\}$ is a set of positive roots. The positive simple roots are then the roots of the form $\alpha_{i}=L_{i}-L_{i+1}$, with $i=1, \ldots, n-1$, and the dual basis of $\mathfrak{t}$ is formed by the matrices

$$
H_{i}=\frac{n-i}{n} E_{1}+\ldots+\frac{n-i}{n} E_{i}-\frac{i}{n} E_{i+1}-\ldots-\frac{i}{n} E_{n} .
$$

The Lie group $\mathrm{SU}(n)$ is precisely the simply connected Lie group with Lie algebra $\mathfrak{s u}(n)$ and its center is $\mathbb{Z}_{n}$. The integer lattice $\mathfrak{I}\left(\mathrm{SU}(n) / \mathbb{Z}_{n}\right)$ is simply $\left\{\sum n_{i} H_{i}: n_{i} \in \mathbb{Z}\right\}$ and its $I$-canonical elements with respect to $\Delta^{+}$are the sums $\sum_{i \in I} H_{i}$, with $I \subseteq\{1, \ldots, n-$ $1\}$. The $I$-canonical elements of $\mathrm{SU}(n)$ are not so easy to identify. We need to find the integral combinations of the elements $H_{i}$ which are in $\mathfrak{I}^{\prime}(\mathrm{G}) \cap \mathfrak{C}_{I}$ (that is, elements which are simultaneously integral combinations of the elements $H_{i}$ and of the elements $E_{i}$ ) and are maximal with respect to $\preceq$. For example, when $n$ is odd, it is easy to check that $\xi=H_{1}+$ 
$H_{2}+\ldots+H_{n-1}$ is the unique $[n-1]$-canonical element of $\mathrm{SU}(n)$ with respect to $\Delta^{+}$, where $[p]=\{1, \ldots, p\}$. But when $n>2$ is even there always exist more than one $[n-1]$-canonical element. The following lemma is useful in order to describe all canonical elements of $\mathrm{SU}(n)$ :

Lemma 5.3. The integer lattice $\mathfrak{I}(\mathrm{SU}(n))$ is invariant with respect to the linear isomorphism $\chi_{1}: \mathfrak{t}^{\mathbb{C}} \rightarrow \mathfrak{t}^{\mathbb{C}}$ defined by $\chi_{1}\left(H_{i}\right)=H_{n-i}$, with $i \in[n-1]$. When $n=2 m+1$ is odd, $\mathfrak{I}(\mathrm{SU}(n))$ is also invariant with respect to the linear isomorphism $\chi_{2}: \mathfrak{t}^{\mathbb{C}} \rightarrow \mathfrak{t}^{\mathbb{C}}$ defined by $\chi_{2}\left(H_{i}\right)=H_{2 i}$ and $\chi_{2}\left(H_{n-i}\right)=H_{n-2 i}$ if $i \in\{1, \ldots, m\}$.

Proof. As we have observed before, an element of $\mathfrak{t}$ is in $\mathfrak{I}(\mathrm{SU}(n))$ if and only if its coefficients in $E_{i}$ are integers. Hence, taking account that $H_{i}=E_{1}+\ldots+E_{i}-\frac{i}{n}\left(E_{1}+\ldots+E_{n}\right)$, an integer linear combination $\sum_{i=1}^{n-1} n_{i} H_{i}$ is in $\mathfrak{I}(\mathrm{SU}(n))$ if and only if $\sum_{i=1}^{n-1} \frac{i n_{i}}{n}$ is an integer number, and this happens if and only if $\sum_{i=1}^{n-1} \frac{(n-i) n_{i}}{n}$ is integer. Hence $\mathfrak{I}(\mathrm{SU}(n))$ is invariant with respect to $\chi_{1}$.

Assume now that $n=2 m+1$. In this case,

$$
\sum_{i=1}^{n-1} \frac{i n_{i}}{n}=\sum_{i=1}^{m} \frac{i n_{i}}{n}+\sum_{i=1}^{m} \frac{(n-i) n_{n-i}}{n}=\sum_{i=1}^{m} \frac{i n_{i}}{n}-\sum_{i=1}^{m} \frac{i n_{n-i}}{n}+\sum_{i=1}^{m} n_{n-i} .
$$

On the other hand, if we set

$$
\sum_{i=1}^{n-1} n_{i}^{\prime} H_{i}=\chi_{2}\left(\sum_{i=1}^{n-1} n_{i} H_{i}\right)=\sum_{i=1}^{m} n_{i} H_{2 i}+\sum_{i=1}^{m} n_{n-i} H_{n-2 i}
$$

we get

$$
\sum_{i=1}^{n-1} \frac{i n_{i}^{\prime}}{n}=\sum_{i=1}^{m} \frac{2 i n_{i}}{n}+\sum_{i=1}^{m} \frac{(n-2 i) n_{n-i}}{n}=2 \sum_{i=1}^{m} \frac{i n_{i}}{n}-2 \sum_{i=1}^{m} \frac{i n_{n-i}}{n}+\sum_{i=1}^{m} n_{n-i} .
$$

Comparing (19) with (20) we conclude that $\sum_{i=1}^{n-1} \frac{i n_{i}^{\prime}}{n} \in \mathbb{Z}$ if $\sum_{i=1}^{n-1} \frac{i n_{i}}{n} \in \mathbb{Z}$, that is $\mathfrak{I}(\mathrm{SU}(n))$ is invariant with respect to $\chi_{2}$.

For each $i \in\{1, \ldots, n-1\}$, let $m_{i}$ be the least positive integer which makes $m_{i} H_{i}$ and integral combination of the elements $E_{i}$. Since $H_{i}=E_{1}+\ldots+E_{i}-\frac{i}{n}\left(E_{1}+\ldots+E_{n}\right), m_{i}$ is precisely the denominator of the irreducible fraction equivalent to $\frac{i}{n}$. The canonical elements should then be sought among the elements of the finite set formed by the integral combinations $\sum_{i=1}^{n-1} n_{i} H_{i}$, with $n_{i} \in\left\{0, \ldots, m_{i}\right\}$, which are simultaneously integral combinations of the elements $E_{i}$. For general $n$ and $I \subseteq\{1, \ldots, n-1\}$ it is too hard to list all the $I$-canonical elements. Next we will describe in detail the situation for the lower dimensional cases. We shall denote by $\pi_{i}$ the orthogonal projection of $\mathbb{C}^{n}$ onto the one-dimensional vector subspace of $\mathbb{C}^{n}$ generated by the vector $e_{i}$.

5.3. The case $\mathrm{SU}(2)$. In this case there is a unique simple root $\alpha_{1}$ with dual $H_{1}=\frac{1}{2}\left(E_{1}-E_{2}\right)$, which does not belong to the integer lattice $\mathfrak{I}(\mathrm{SU}(2))$. Consequently $\xi=2 H_{1}$ is the unique non-trivial canonical element - the corresponding homomorphism is $\gamma_{\xi}(\lambda)=\lambda^{-1} \pi_{1}+\lambda \pi_{1}^{\perp}$. If $W: M \backslash D \rightarrow U_{\xi}(\mathrm{SU}(n))$ is a complex extended solution, then the corresponding $\mathrm{S}^{1}$-invariant solution is given by $u_{\xi}(W)=\lambda^{-1} A+A+\lambda H_{+}^{2}$, where $A$ is a holomorphic subbundle of $\underline{\mathbb{C}}^{2}$. It follows from the super-horizontality property that $A$ is a constant bundle. Hence, we have $W=L+A+\lambda H_{+}^{2}$, where $L$ is a holomorphic line bundle of $A \lambda^{-1}+A^{\perp}$, with $p_{-1}(L) \neq 0$ off 
a discrete set of points, where $p_{-1}$ is defined as in (44). That is, any harmonic map of finite uniton number from $M$ into $\mathrm{SU}(2) \simeq \mathrm{S}^{3}$ arises from a constant direction $A$ and a holomorphic line bundle of $\lambda^{-1} A+A^{\perp} \simeq \underline{\mathbb{C}}^{2}$. This agrees with the well known result by Calabi 4 that asserts that any locally minimal immersion of a surface in an odd dimensional sphere $S^{2 m-1}$ is contained in a hyperplane of $\mathbb{R}^{2 m}$. In particular, no harmonic map of finite uniton number from $M$ into $\mathrm{SU}(2) \simeq \mathrm{S}^{3}$ is full. This means that any such harmonic map takes values in a unit two-dimensional sphere $\mathrm{S}^{2} \simeq \mathbb{C P}^{1}$, that is, it corresponds to an holomorphic line bundle of $\underline{\mathbb{C}}^{2}$.

5.4. The case $\mathrm{SU}(3)$. We have two simple roots, $\alpha_{1}$ and $\alpha_{2}$, and three non-trivial canonical elements:

$$
\xi_{1}=H_{1}+H_{2}=E_{1}-E_{3} ; \quad \xi_{2}=3 H_{1}=2 E_{1}-E_{2}-E_{3} ; \quad \xi_{3}=3 H_{2}=E_{1}+E_{2}-2 E_{3} .
$$

The corresponding homomorphisms are given by

$$
\gamma_{\xi_{1}}(\lambda)=\lambda^{-1} \pi_{3}+\pi_{2}+\lambda \pi_{1} ; \quad \gamma_{\xi_{2}}(\lambda)=\lambda^{-1}\left(\pi_{2}+\pi_{3}\right)+\lambda^{2} \pi_{1} ; \quad \gamma_{\xi_{3}}(\lambda)=\lambda^{-2} \pi_{3}+\lambda\left(\pi_{2}+\pi_{1}\right) .
$$

If $W_{\xi_{1}}: M \backslash D \rightarrow U_{\xi_{1}}(\mathrm{SU}(3))$ is a complex extended solution, then the corresponding $\mathrm{S}^{1}$ invariant solution is given by $u_{\xi_{1}}\left(W_{\xi_{1}}\right)=\lambda^{-1} B_{3}+\left(B_{2} \oplus B_{3}\right)+\lambda H_{+}^{3}$, where $B_{3}$ is a holomorphic line subbundle of the holomorphic vector bundle $B_{2} \oplus B_{3}$ of rank 2 . In order to construct all such extended solutions, and taking account the results of section 4.5, we start with a meromorphic section $s_{3}$ of $\underline{\mathbb{C}}^{3}$ and set $B_{3}=\operatorname{Span}\left\{s_{3}\right\}$. If $B_{3}$ is not constant, we define $B_{2}=B_{3}^{(1)} \ominus B_{3}$, take an arbitrary holomorphic section $w_{3}$ of $\mathbb{C}^{3}$ and set $X_{\xi_{1}}=\operatorname{Span}\left\{\lambda^{-1} s_{3}+w_{3}\right\}$. If $B_{3}$ is constant, we take an arbitrary meromorphic section $s_{2}$. By adding a constant if necessary, $s_{2}$ and $s_{3}$ are linearly independent and we set $B_{2}=\operatorname{Span}\left\{s_{2}, s_{3}\right\} \ominus B_{3}$. Take an arbitrary holomorphic section $w_{3}$ of $\underline{\mathbb{C}}^{3}$ and set $X_{\xi_{1}}=\operatorname{Span}\left\{\lambda^{-1} s_{3}+w_{3}, s_{2}\right\}$. In both cases, $X_{\xi_{1}}$ is a Frenet frame for an extended solution $W_{\xi_{1}}: M \backslash D \rightarrow U_{\xi_{1}}(\mathrm{SU}(3))$.

If $W_{\xi_{2}}: M \backslash D \rightarrow U_{\xi_{2}}(\mathrm{SU}(3))$ is a complex extended solution, then the corresponding $\mathrm{S}^{1}$ invariant solution is given by

$$
u_{\xi_{2}}\left(W_{\xi_{2}}\right)=\lambda^{-1}\left(B_{2} \oplus B_{3}\right)+\left(B_{2} \oplus B_{3}\right)+\lambda\left(B_{2} \oplus B_{3}\right)+\lambda^{2} H_{+}^{3} .
$$

By the super-horizontality property, $B_{2} \oplus B_{3}$ is constant, and consequently $B_{1}$, the orthogonal complement of $A_{2} \oplus A_{3}$, is also constant. In order to construct all such extended solutions, fix a two-dimensional subspace with basis elements $s_{2}$ and $s_{3}$. Take arbitrary meromorphic sections $w_{2}$ and $w_{3}$ of $\mathbb{C}^{3}+\lambda \underline{\mathbb{C}}^{3}$ with $\pi_{B_{1}}\left(p_{0}\left(w_{2}\right)\right)$ and $\pi_{B_{1}}\left(p_{0}\left(w_{3}\right)\right)$ constants, where $p_{0}$ is the projection defined by (44). Then $X_{\xi_{2}}=\operatorname{Span}\left\{s_{2} \lambda^{-1}+w_{2}, s_{3} \lambda^{-1}+w_{3}\right\}$ is a Frenet frame for an extended solution $W_{\xi_{2}}: M \backslash D \rightarrow U_{\xi_{2}}(\mathrm{SU}(3))$, and all such extended solutions arise in this way. We observe that, taking account Lemma 3.17 and Proposition 3.18 of [13], the corresponding harmonic map has uniton number one, in the sense that it admits an extended solution with values in $\Omega \mathrm{U}(3)$ of the form $\pi_{V}+\lambda \pi_{V}^{\perp}$, with $V$ a holomorphic subbundle of $\mathbb{C}^{3}$. The case $W_{\xi_{3}}$ is similar.

5.5. The cases $\mathrm{SU}(4)$ and $\mathrm{SU}(5)$. Table 1 shows all the non-trivial canonical elements of $\mathrm{SU}(4)$ and $\mathrm{SU}(5)$ up to the symmetries $\chi_{1}, \chi_{2}$ of Lemma 5.3 .

We describe how to construct, for $\xi_{1}=H_{1}+2 H_{2}+H_{3}=2 E_{1}+E_{2}-E_{3}-2 E_{4}$, all the extended solutions $W_{\xi_{1}}: M \backslash D \rightarrow U_{\xi_{1}}(\mathrm{SU}(4))$. We have $\gamma_{\xi_{1}}=\lambda^{-2} \pi_{4}+\lambda^{-1} \pi_{3}+\lambda \pi_{2}+\lambda^{2} \pi_{1}$, and, consequently,

$$
u_{\xi_{1}}\left(W_{\xi_{1}}\right)=\lambda^{-2} B_{4}^{1}+\lambda^{-1}\left(B_{3}^{1} \oplus B_{4}^{1}\right)+\left(B_{3}^{1} \oplus B_{4}^{1}\right)+\lambda\left(B_{2}^{1} \oplus B_{3}^{1} \oplus B_{4}^{1}\right)+\lambda^{2} H_{+}^{4},
$$




\begin{tabular}{c|c|c|c|c}
$\mathrm{SU}(n)$ & $|I|=n-1$ & $|I|=n-2$ & $|I|=n-3$ & $|I|=n-4$ \\
\hline$n=4$ & $H_{1}+2 H_{2}+H_{3}$ & $2 H_{1}+H_{2}$ & $4 H_{1}$ & \\
& $3 H_{1}+H_{2}+H_{3}$ & $H_{1}+H_{3}$ & $2 H_{2}$ & \\
\hline$n=5$ & $H_{1}+H_{2}+H_{3}+H_{4}$ & $H_{1}+H_{2}+4 H_{3}$ & $H_{1}+2 H_{2}$ & $5 H_{1}$ \\
& & $H_{1}+3 H_{2}+H_{3}$ & $3 H_{1}+H_{2}$ & \\
& & $2 H_{1}+H_{2}+2 H_{3}$ & $H_{1}+H_{4}$ & \\
& & $3 H_{1}+2 H_{2}+H_{3}$ & & \\
& & $5 H_{1}+H_{2}+H_{3}$ & &
\end{tabular}

TABLE 1. Canonical elements for SU(4) and SU(5).

where each vector subbundle $B_{i}^{1}$ has rank one. The harmonic map associated to this $\mathrm{S}^{1}$-invariant extended solution is given by

$$
\varphi_{1}=\pi_{B_{1}^{1} \oplus B_{4}^{1}}-\pi_{B_{2}^{1} \oplus B_{3}^{1}} .
$$

By super-horizontality, $B_{3}^{1} \oplus B_{4}^{1}$ is a constant bundle. So, in order to construct all such extended solutions, we start by fixing a two-dimensional vector subspace $V$ of $\mathbb{C}^{4}$ generated by constant vectors $u$ and $v$. Next take meromorphic sections:

(1) $s_{1}$ of $\underline{V}$, and set $B_{4}^{1}=\operatorname{Span}\left\{s_{1}\right\}$ and $B_{3}^{1}=\underline{V} \ominus B_{4}^{1}$;

(2) $s_{3}$ of $\underline{V}^{\perp}$, and set $B_{2}^{1}=\operatorname{Span}\left\{s_{3}\right\}$ and $B_{1}^{1}=\underline{V}^{\perp} \ominus B_{2}^{1}$;

(3) $w_{1}$ of $M \times H_{+}^{4} / \lambda^{3} H_{+}^{4}$.

If $B_{4}^{1}$ is not constant, we can write $s_{1}^{\prime \prime}=g_{1} s_{1}+g_{2} s_{1}^{\prime}$ for some meromorphic functions $g_{1}$ and $g_{2}$ on $M$, with $g_{1}^{\prime} g_{2}-g_{2}^{\prime} g_{1} \neq 0$. In this case, $X=\operatorname{Span}\left\{\lambda^{-2} s_{1}+\lambda^{-1} w_{1}, \lambda s_{3}\right\}$ is a Frenet frame for an extended solution with values in $U_{\xi_{1}}(\mathrm{SU}(4))$ if and only if

$$
\pi_{B_{1}^{1}} \circ p_{0}\left(w_{1}^{\prime \prime}-g_{1} w_{1}-g_{2} w_{1}^{\prime}\right)=0 .
$$

For $\xi_{2}=3 H_{1}+H_{2}+H_{3}=3 E_{1}-E_{3}-2 E_{4}$, we have $\gamma_{\xi_{2}}=\lambda^{-2} \pi_{4}+\lambda^{-1} \pi_{3}+\pi_{2}+\lambda^{3} \pi_{1}$, and, consequently,

$$
\begin{aligned}
u_{\xi_{2}}\left(W_{\xi_{2}}\right)=\lambda^{-2} B_{4}^{2} & +\lambda^{-1}\left(B_{3}^{2} \oplus B_{4}^{2}\right) \\
& +\left(B_{2}^{2} \oplus B_{3}^{2} \oplus B_{4}^{2}\right)+\lambda\left(B_{2}^{2} \oplus B_{3}^{2} \oplus B_{4}^{2}\right)+\lambda^{2}\left(B_{2}^{2} \oplus B_{3}^{2} \oplus B_{4}^{2}\right)+\lambda^{3} H_{+}^{4} .
\end{aligned}
$$

The harmonic map associated to this $\mathrm{S}^{1}$-invariant extended solution is given by

$$
\varphi_{2}=\pi_{B_{2}^{2} \oplus B_{4}^{2}}-\pi_{B_{1}^{2} \oplus B_{3}^{2}} .
$$

Although $\varphi_{1}$ and $\varphi_{2}$ are both of the form $\pi_{E}-\pi_{E}^{\perp}$, with $E$ a rank two vector subbundle of $\underline{\mathbb{C}}^{4}$, these vector bundles exhibit distinct geometrical behaviours. For example, whereas $E=B_{2}^{2} \oplus B_{4}^{2}$ has always constant (2)-osculating bundle, both $E=B_{1}^{1} \oplus B_{4}^{1}$ and $E^{\perp}=B_{2}^{1} \oplus B_{3}^{1}$ can have non-constant (2)-osculating bundle.

5.6. Symmetric canonical elements of $\mathrm{SU}(n)$. All the compact inner symmetric spaces of $\mathrm{SU}(n)$ are complex grassmannians $\operatorname{Gr}(k, n)$. The embedding $\iota$ of $\operatorname{Gr}(k, n)$ as a connected component of $\sqrt{e}$ is given by $\iota(V)=\pi_{V}-\pi_{V}^{\perp}$, where $V \in \operatorname{Gr}(k, n)$ is $k$-dimensional subspace of $\mathbb{C}^{n}$. There exists no non-trivial symmetric canonical element for $\mathrm{SU}(2)$. Table 2 presents all non-trivial symmetric canonical elements for $\mathrm{SU}(n)$, with $n=3,4,5$, up to the symmetries $\chi_{1}, \chi_{2}$. As before, for each $i \in\{1, \ldots, n-1\}$, let $m_{i}$ be the least positive integer which 
makes $m_{i} H_{i}$ and integral combination of the elements $E_{i}$. The symmetric canonical elements should then be sought among the elements of the finite set formed by the integral combinations $\sum_{i=1}^{n-1} n_{i} H_{i}$, with $n_{i} \in\left\{0, \ldots, 2 m_{i}-1\right\}$, which are simultaneously integral combinations of the elements $E_{i}$.

We use the usual hermitian inner product on $\mathbb{C}^{n}$ to identify $\operatorname{Gr}(k, n)$ with $\operatorname{Gr}(n-k, n)$. It is easy to check that, for $\xi \in \mathfrak{I}(\mathrm{SU}(n)), N_{\xi}=N_{\chi_{1}(\xi)}$. However, in general, the symmetric space $N_{\chi_{2}(\xi)}$ does not coincide with $N_{\xi}$. For example, in $\mathrm{SU}(5)$ the two following situations can occur: for $\xi=5 H_{1}$, we have $\chi_{2}(\xi)=5 H_{2}, N_{\xi}=\operatorname{Gr}(1,5)$ and $N_{\chi_{2}(\xi)}=\operatorname{Gr}(2,5)$; on the other hand, for $\eta=3 H_{1}+H_{2}+5 H_{3}$, we have $N_{\eta}=N_{\chi_{2}(\eta)}=\operatorname{Gr}(2,5)$.

\begin{tabular}{c|c|c|c|c}
$\operatorname{Gr}(k, n)$ & $|I|=n-1$ & $|I|=n-2$ & $|I|=n-3$ & $|I|=n-4$ \\
\hline$k=1, n=3$ & $H_{1}+H_{2}$ & $3 H_{1}$ & & \\
& $4 H_{1}+H_{2}$ & & & \\
\hline$k=2, n=4$ & $3 H_{1}+H_{2}+H_{3}$ & $2 H_{1}+H_{2}$ & & \\
\hline$k=1, n=5$ & $4 H_{1}+2 H_{2}+H_{3}+H_{4}$ & $H_{1}+H_{2}+4 H_{3}$ & $H_{1}+2 H_{2}$ & $5 H_{1}$ \\
& & & $H_{1}+7 H_{2}$ & \\
& & & $3 H_{1}+H_{2}$ & \\
\hline$k=2, n=5$ & $H_{1}+H_{2}+H_{3}+H_{4}$ & $H_{1}+H_{2}+9 H_{3}$ & $4 H_{1}+3 H_{2}$ & \\
& $2 H_{1}+3 H_{2}+H_{3}+H_{4}$ & $H_{1}+3 H_{2}+H_{3}$ & $8 H_{1}+H_{2}$ & \\
& $H_{1}+H_{2}+H_{3}+6 H_{4}$ & $H_{1}+8 H_{2}+H_{3}$ & $H_{1}+H_{4}$ & \\
& & $2 H_{1}+H_{2}+2 H_{3}$ & & \\
& & $3 H_{1}+H_{2}+5 H_{3}$ & & \\
& & $3 H_{1}+2 H_{2}+H_{3}$ & & \\
& & $5 H_{1}+H_{2}+H_{3}$ & &
\end{tabular}

TABLE 2. Symmetric canonical elements for $\mathrm{SU}(n)$, with $n \leq 5$.

We describe how to construct, for $n=4, k=2$ and $\xi_{1}=2 H_{1}+H_{2}=2 E_{1}-E_{3}-E_{4}$, all the extended solutions $W_{\xi_{1}}: M \backslash D \rightarrow U_{\xi_{1}}^{\mathcal{I}}(\mathrm{SU}(4))$. We have $\gamma_{\xi_{1}}=\lambda^{-1}\left(\pi_{3}+\pi_{4}\right)+\pi_{2}+\lambda^{2} \pi_{1}$ and, consequently,

$$
u_{\xi_{1}}\left(W_{\xi_{1}}\right)=\lambda^{-2}\left(B_{4}^{1} \oplus B_{3}^{1}\right)+\left(B_{4}^{1} \oplus B_{3}^{1} \oplus B_{2}^{1}\right)+\lambda\left(B_{4}^{1} \oplus B_{3}^{1} \oplus B_{2}^{1}\right)+\lambda^{2} H_{+}^{4},
$$

where each vector subbundle $B_{i}^{1}$ has rank one. The harmonic map associated to this $\mathrm{S}^{1}$ invariant extended solution is given by $\varphi_{1}=\pi_{B_{1}^{1} \oplus B_{2}^{1}}-\pi_{B_{3}^{1} \oplus B_{4}^{1}}$. So, take meromorphic sections $s_{1}, s_{2}, w_{1}, w_{2}$ of $\mathbb{C}^{4}$ and set $B_{3} \oplus B_{4}=\operatorname{Span}\left\{s_{1}, s_{2}\right\}$. Assuming that this vector bundle is not constant, $X_{\xi_{1}}=\operatorname{Span}\left\{\lambda^{-1} s_{1}+\lambda w_{1}, \lambda^{-1} s_{2}+\lambda w_{2}\right\}$ will be a Frenet frame for an extended solution with values in $U_{\xi_{1}}^{\mathcal{I}}(\mathrm{SU}(4))$. Moreover, all such extended solutions, with $B_{3} \oplus B_{4}$ not constant, arise in this way.

Consider also the case $n=4, k=2$ and $\xi_{2}=H_{1}+H_{3}=E_{1}-E_{4}$. We have $\gamma_{\xi_{2}}=$ $\lambda^{-1} \pi_{4}+\left(\pi_{3} \oplus \pi_{2}\right)+\lambda \pi_{1}$ and, consequently, $u_{\xi_{2}}\left(W_{\xi_{2}}\right)=\lambda^{-1} B_{4}^{2}+B_{4}^{2} \oplus B_{3}^{2} \oplus B_{2}^{2}+\lambda H_{+}^{4}$. The harmonic map associated to this $\mathrm{S}^{1}$-invariant extended solution is given by $\varphi_{2}=\pi_{B_{2}^{2} \oplus B_{3}^{2}}-\pi_{B_{1}^{2} \oplus B_{4}^{2}}$. Observe that, in this case, we only have $S^{1}$-invariant extended solutions since $U_{\xi_{2}}^{\mathcal{I}}(\mathrm{SU}(4))=\Omega_{\xi_{2}}$. 


\section{REFERENCES}

[1] F.E. Burstall and M.A. Guest, Harmonic Two-Spheres in Compact Symmetric Spaces, revisited, Math. Ann. 309 (1997), no. 4, 541-572.

[2] F.E Burstall and J.H. Rawnsley, Twistor Theory for Riemannian Symmetric Spaces with Applications to Harmonic Maps of Riemann Surfaces, Lecture Notes in Mathematics, no. 1424, Springer-Verlag, Berlin, Heidelberg, 1990.

[3] F.E Burstall and J.C. Wood, The Construction of Harmonic Maps into Complex Grassmannians, J. Differential Geom. 23 (1986), no. 3, 255-297.

[4] E. Calabi, Minimal Immersions of Surfaces in Euclidean Spheres, J. Differential Geom. 1 (1967), 111-125.

[5] N. Correia and R. Pacheco, Singular Dressing Actions on Harmonic Maps, Q. J. Math. 62 (2011), no. 1, 71-85.

[6] - Harmonic Maps of Finite Uniton Number into $\mathrm{G}_{2}$, Math. Z. 271 (2012), no. 1-2, 13-32.

[7] J. Eells and L. Lemaire, Another report on harmonic maps, Bull. Lond. Math. Soc. 20 (1988).

[8] M.J. Ferreira, B.A. Simões, and J.C. Wood, All harmonic 2-spheres in the unitary group, completly explicitly, Math. Zeit. 266 (2010), 953-978.

[9] M.A. Guest, An update on Harmonic Maps of Finite Uniton Number, via the Zero Curvature Equation, Integrable Systems, Topology, and Physics: A Conference on Integrable Systems in Differential Geometry (M. Guest, R. Miyaoka, and Y. Ohnita, eds.), Contemporary Mathematics, no. 309, Amer. Math. Soc., 2002, pp. 85-113.

[10] R. Pacheco, Harmonic Two-Spheres in the Sympletic Group $\operatorname{Sp}(n)$, Internat. J. Math. 17 (2006), no. 3, 295-311.

[11] A. Pressley and G. Segal, Loop Groups, Oxford Mathematical Monographs, 2003.

[12] G. Segal, Loop Groups and Harmonic Maps, Advances in Homotopy Theory (S.M. Salamon, B. Steer, and W.A. Sutherland, eds.), Lecture Note Series, no. 139, London Mathematical Society, 1989, pp. 153-164.

[13] M. Svensson and J.C. Wood, Filtrations, Factorizations and Explicit Formulae for Harmonic Maps, Comm. Math. Phys. 310 (2012), no. 1, 99-134.

[14] K. Uhlenbeck, Harmonic Maps into Lie Groups (classical solutions of the chiral model), J. Differential Geom. 30 (1989), no. 1, 1-50.

Universidade da Beira Interior, Rua Marques d’Avila e Bolama, 6200-001 Covilha, Portugal E-mail address: ncorreia@ubi.pt

Universidade da Beira Interior, Rua Marques d’Avila e Bolama, 6200-001 Covilha, Portugal E-mail address: rpacheco@ubi.pt 